OPEN ACCESS

Edited by: Alba Di Leone,

Agostino Gemelli University Polyclinic (IRCCS), Italy

Reviewed by:

Chukwuemeka Ihemelandu, MedStar Washington Hospital Center,

United States

Giulia Turri,

University of Verona, Italy

${ }^{*}$ Correspondence:

Melissa Ching Ching Teo melissa.teo.c.c@singhealth.com.sg

${ }^{\dagger}$ These authors have contributed equally to this work and share first authorship

Specialty section: This article was submitted to

Surgical Oncology,

a section of the journal

Frontiers in Oncology

Received: 09 November 2021 Accepted: 15 December 2021

Published: 13 January 2022

Citation:

Wong JSM, Lek SM, Lim DYZ, Chia CS, Tan GHC, Ong C-AJ and

Teo MCC (2022) Palliative Gastrointestinal Surgery in Patients With Advanced Peritoneal Carcinomatosis: Clinical Experience and Development of a Predictive Model for Surgical Outcomes.

Front. Oncol. 11:811743. doi: 10.3389/fonc.2021.811743

\section{Palliative Gastrointestinal Surgery in Patients With Advanced Peritoneal Carcinomatosis: Clinical Experience and Development of a Predictive Model for Surgical Outcomes}

\author{
Jolene Si Min Wong ${ }^{1,2,3,4 t}$, Sze Min Lek ${ }^{5 \dagger}$, Daniel Yan Zheng Lim ${ }^{6}$, \\ Claramae Shulyn Chia ${ }^{1,2,3,4}$, Grace Hwei Ching Tan ${ }^{1,2}$, Chin-Ann Johnny Ong 1,2,3,4,7,8 \\ and Melissa Ching Ching Teo ${ }^{1,2,3,4 *}$

\begin{abstract}
Department of Sarcoma, Peritoneal and Rare Tumours (SPRinT), Division of Surgery and Surgical Oncology, National Cancer Centre Singapore, Singapore, Singapore, ${ }^{2}$ Department of Sarcoma, Peritoneal and Rare Tumours (SPRinT), Division of Surgery and Surgical Oncology, Singapore General Hospital, Singapore, Singapore, ${ }^{3}$ SingHealth Duke-NUS Surgery Academic Clinical Program, Duke-NUS Medical School, Singapore, Singapore, ${ }^{4}$ SingHealth Duke-NUS Oncology Academic Clinical Program, Duke-NUS Medical School, Singapore, Singapore, ${ }^{5}$ Department of Anaesthesia and Surgical Intensive Care, Changi General Hospital, Singapore, Singapore, ${ }^{6}$ Health Services Research Unit, Medical Board, Singapore General Hospital, Singapore, Singapore, ${ }^{7}$ Laboratory of Applied Human Genetics, Division of Medical Sciences, National Cancer Centre
\end{abstract} \\ Singapore, Singapore, Singapore, ${ }^{8}$ Institute of Molecular and Cell Biology, ASTAR Research Entities, Singapore, Singapore
}

Background: Palliative gastrointestinal (Gl) surgery potentially relieves distressing symptoms arising from intestinal obstruction $(\mathrm{IO})$ in patients with advanced peritoneal carcinomatosis $(\mathrm{PC})$. As surgery is associated with significant morbidity risks in advanced cancer patients, it is important for surgeons to select patients who can benefit the most from this approach. Hence, we aim to determine predictors of morbidity and mortality after palliative surgery in patients with PC. In addition, we evaluate the utility of the UC Davis Cancer Care nomogram (UCDCCn) and develop a simplified model to predict short-term surgical mortality in these patients.

Methods: A retrospective review of patients with $1 \mathrm{O}$ secondary to PC undergoing palliative Gl surgery was performed. Logistic regression was used to determine independent predictors of 30-day morbidity and mortality after surgery. UCDCCn was evaluated using the area under the curve (AUC) for discriminatory power and the HosmerLemeshow test for calibration. Our simplified model was developed using logistic regression and evaluated using cross-validation.

Results: A total of 254 palliative Gl surgeries were performed over a 10-year duration. The 30-day morbidity and mortality were $43 \%(n=110)$ and $21 \%(n=53)$, respectively. Preoperative albumin, age, and emergency nature of surgery were significant independent predictors for 30-day morbidity. A simplified model using preoperative Eastern Cooperative Oncology Group (ECOG) status and albumin (AUC = 0.71) achieved better predictive power than UCDCCn (AUC $=0.66$ ) for 30-day mortality. 
Conclusion: Good ECOG status and high preoperative albumin levels were independently associated with good short-term outcomes after palliative Gl surgery. Our simplified model may be used to conveniently and efficiently select patients who stand to benefit the most from surgery.

Keywords: advanced cancer, intestinal obstruction, palliation, palliative surgery, peritoneal carcinomatosis

\section{INTRODUCTION}

Peritoneal carcinomatosis (PC) is an end-stage presentation of up to $50 \%$ of advanced cancer patients with various primary tumors $(1,2)$. Debilitating gastrointestinal (GI) symptoms due to complex, multilevel intestinal obstruction (IO) are common and may not be adequately palliated with medical or endoscopic therapy alone (3). As such, though infrequently publicized, palliative surgeries make up approximately one-fifth of all surgical procedures performed at any major cancer center annually (4). In fact, most report high rates of clinical success ranging from $80 \%$ to $100 \%$ after palliative GI surgery for PCassociated IO $(5,6)$.

Though a direct and effective means of palliation in IO, surgery is associated with significant morbidity risks. A systematic review of 17 retrospective studies on surgical management of malignant bowel obstruction found that serious complications occurred in up to $44 \%$ of patients, and mortality rates ranged from $6 \%$ to $32 \%$ (5). Citing high morbidities and in-hospital deaths among advanced cancer patients undergoing palliative surgery, some physicians adopt a blanket "no surgery" approach in favor of medical treatment alone among palliative PC patients $(7,8)$. This misconception deprives suitable surgical candidates of a treatment modality that can provide good palliation during end of life. As such, there is a need to identify palliative PC patients who will benefit the most from surgery while adopting discretion when offering a surgical mode of palliation in those in whom poor outcomes are expected.

The UC Davis nomogram was developed to predict 30-day morbidity and mortality among patients with disseminated malignancy who had undergone surgical intervention (9). With the use of data from the American College of Surgeons National Surgical Quality Improvement Program (ACS NSQIP), preoperative factors affecting an individual's risk of perioperative morbidity and mortality were identified. Thirteen and fourteen factors including "do not resuscitate (DNR)" status, age, weight loss $>10 \%$, dyspnea, functional dependence, ascites, chronic steroid use, active sepsis, serum creatinine level, serum albumin level, serum white blood cell (WBC), serum hematocrit and acuity of surgical procedure, and procedure type were found to be independently associated with postoperative complications and death, respectively. With the use of the above factors, nomograms predictive of the probability of experiencing a postoperative event were then constructed. While comprehensive, the model has not been independently validated and may be cumbersome when applied in clinical practice due to its complexity.
As such, our study aims to report our clinical experience in palliative GI surgery in the context of PC and evaluate the utility of the UC Davis model in predicting perioperative outcomes in our patient cohort. We also aim to develop a simplified model to predict 30-day morbidity and mortality among patients undergoing palliative surgery.

\section{MATERIALS AND METHODS}

A retrospective review of $\mathrm{PC}$ patients who underwent palliative surgery for IO at the Singapore General Hospital was performed from January 2009 to January 2019. Patients with PC from a variety of primary malignancies, including GI, gynecological, hepato-pancreatico-biliary, and others, were included. Patient demographics, perioperative variables, tumor characteristics, and postoperative morbidity and mortality outcomes were obtained from medical records.

The study was conducted with the approval of the ethics board.

\subsection{Definitions}

\subsubsection{Peritoneal Carcinomatosis and Intestinal Obstruction}

All patients had a histologically proven diagnosis of malignancy and histologically or radiologically proven metastases, specifically metastases to the peritoneum, at the time of surgery. IO was defined clinically based on signs and symptoms of obstruction such as abdominal distention, abdominal pain, nausea and vomiting, constipation, inability to pass air, or radiologically on imaging modalities performed (10).

\subsubsection{Preoperative, Intraoperative, and Postoperative Variables}

The following comorbid conditions were determined to be absent or present based on ACS NSQIP criteria (11). Dyspnea was defined as the presence of labored breathing on exertion or at rest. Significant weight loss was defined as weight loss of $10 \%$ in the previous 6 months. Preoperative sepsis was defined as a positive bacterial culture identified in addition to two or more of the following criteria: fever, tachycardia, tachypnea, leukocytosis, and anion gap acidosis. Preoperative chemotherapy or radiotherapy was defined as the administration of chemotherapy within 30 days and radiotherapy within 90 days before surgery.

Intraoperatively, the type of surgical procedures was stratified to consider if GI resection, multi-visceral resection, and other abdominal surgical procedures such as adhesiolysis were 
performed as per the ACS NSQIP classification. We further collected information on the type of bowel resection, anastomoses, and stoma fashioned. Emergency cases were designated by the primary surgeon after considering the clinical circumstances surrounding palliative surgical interventions.

Data on postoperative complications were collected and included organ-specific complications (hematological, cardiac, respiratory, neurologic, abdominal, and others). This was in line with ACS NSQIP-reported complication codes. Unplanned readmissions and Calvien-Dindo-based classification of major and minor postoperative complications were recorded as well (12).

\subsubsection{0-Day Overall Morbidity and Mortality}

Morbidity and mortality were considered at 30 days calculated from the date of palliative surgery.

\subsection{Statistical Analysis}

The baseline statistics of the cohort were summarized with a mean (SD) for continuous variables and N (\%) for categorical variables. Univariate statistical testing was performed for significant associations between individual preoperative and postoperative variables, with 30-day mortality and 30-day morbidity. We used t-test for continuous variables and chisquare testing for categorical variables.

For the development of the multivariate and simplified multivariate models, the data were split into training and test sets in a 7:3 ratio. Continuous variables were scaled and normalized. Multivariate logistic regression was performed on the training set, with preoperative variables used as predictive factors. The backward method of multivariate logistic regression was used in view of the large number of potential predictors identified. To evaluate discriminative power, the area under the curve (AUC) was evaluated on the test set, with the confidence limits determined by bootstrapping. Sensitivity and specificity were calculated using Youden's method to determine the optimal cutoff point.

UC Davis 30-day morbidity and mortality predicted probabilities were calculated from the UC Davis Nomogram. The AUC was used to determine its discriminatory power and the Hosmer-Lemeshow test (H-L test) for calibration. 95\% CIs for the AUC were determined via bootstrapping.

Statistical analysis was performed using the Statistical Package for Social Sciences version 24 (SPSS Inc., Chicago, IL, USA), R version 3.6.1, and Python 3.7. Statistical significance was defined at the 0.05 level.

\section{RESULTS}

\subsection{Baseline Characteristics}

A total of 254 palliative GI surgeries were performed among PC patients over a 10-year duration. The median age of our patients was 61.5 (range 52-71). The most common site of primary malignancy was the colon (42.6\%). All patients had radiographic or grossly seen peritoneal disease, which was subsequently confirmed on histopathologic specimens; $24.4 \%$ had lung metastases, and $31.8 \%$ had liver metastases in addition to peritoneal metastases. The demographic and clinical characteristics of the patients are presented in Table $\mathbf{1}$.

\subsection{Predictors of 30-Day Morbidity and Mortality}

The 30-day morbidity after palliative GI surgery was $43 \%$ ( $\mathrm{n}=$ 110). The most common complications included hematologic complications $31.1 \%$ (i.e., requiring multiple blood product transfusions), intra-abdominal complications 29.5\% (i.e., intraabdominal sepsis and collections), respiratory complications $22.0 \%$, wound $18.1 \%$, and cardiac $14.1 \%$ complications. Minor (Calvien-Dindo grades 1 and 2) and major (Grade 3 onwards) complications occurred in $43 \%$ and $57 \%$ of patients, respectively. Of the patients, $20.1 \%$ and $5.9 \%$ had unplanned readmissions and unplanned reoperations, respectively.

Low preoperative albumin and hematocrit, dyspnea, preoperative use of steroids, and preoperative sepsis were predictors of 30-day morbidity on univariate analysis (Table 2). On multivariate analysis, preoperative albumin, age, and emergency nature of surgery were found to be independent significant predictors with an AUC of 0.62 (95\% CI 0.50 0.76 , Figure 1).

The 30 -day mortality was $21 \%(n=53)$. As all patients had advanced cancer with a prognosis of less than 1 year, it was found that $81 \%(n=206)$ demised within 1 year of palliative surgical intervention. Median survival was 109 days (range 43-265).

The presence of ascites, high Eastern Cooperative Oncology Group (ECOG) status, low albumin, and extent of surgery were associated with higher 30-day mortality on univariate analysis (Table 2). Patients with ovarian primaries had a significantly lowered risk of death at 30 days $(p=0.004)$. On multivariate logistic regression analysis with backward variable selection, only ECOG status and preoperative albumin levels were found to be independent significant predictors (Table 3 ). The final multivariable model had an AUC of 0.77 (95\% CI 0.64-0.90, Figure 1) and sensitivity and specificity of 0.76 and 0.75 , respectively.

\subsection{Utility of the UC Davis Nomogram in Our Patient Cohort}

In the prediction of 30-day morbidity, the UC Davis Nomogram had an AUC of 0.62 (95\% CI 0.55-0.69), and the H-L test had a p-value of 0.99 . This indicated poor discriminative power but acceptable calibration (Figure 2).

In the prediction of 30-day mortality, the UC Davis Nomogram had an AUC of 0.66 (95\% CI 0.57-0.75) for 30day mortality, indicating poor discriminative power. The $\mathrm{H}-\mathrm{L}$ test had a $\mathrm{p}$-value $<0.05$, indicating poor calibration.

\subsection{Simplified Model for 30-Day Morbidity and Mortality Outcomes for Palliative Gastrointestinal Surgery Patients}

To develop a simplified model, we entered the significant variables of ECOG and preoperative albumin found on 
TABLE 1 | Demographics and clinical characteristics of palliative Gl surgery patients.

\begin{tabular}{|c|c|}
\hline Variable & Mean or $\mathbf{N}(\%)$ \\
\hline Age & $61.5(52.3-71)$ \\
\hline Male & $101(40 \%)$ \\
\hline Smoker & $10(4 \%)$ \\
\hline \multicolumn{2}{|l|}{ Site of primary malignancy } \\
\hline Lung & $7(3 \%)$ \\
\hline Stomach & 32 (13\%) \\
\hline Pancreas & $14(6 \%)$ \\
\hline Colon & 108 (43\%) \\
\hline Ovary & $33(13 \%)$ \\
\hline Endometrial & $6(2 \%)$ \\
\hline Cervix & $5(2 \%)$ \\
\hline Others & 49 (19\%) \\
\hline Presence of lung metastases & $62(24 \%)$ \\
\hline Presence of liver metastases & 81 (32\%) \\
\hline \multicolumn{2}{|l|}{ Comorbid disease } \\
\hline Hypertension (requiring medication) & 89 (35\%) \\
\hline Diabetes (requiring medication) & 49 (19\%) \\
\hline Chronic obstructive pulmonary disease & $3(1 \%)$ \\
\hline Myocardial infarction & $5(2 \%)$ \\
\hline Congestive heart failure & $4(2 \%)$ \\
\hline Peripheral vascular disease & $1(0 \%)$ \\
\hline Renal failure & $7(3 \%)$ \\
\hline Dialysis & $4(2 \%)$ \\
\hline \multicolumn{2}{|l|}{ Preoperative clinical characteristics } \\
\hline Emergency surgery & $172(68 \%)$ \\
\hline Prehospital location (home) & 249 (98\%) \\
\hline Independent functional status & 246 (97\%) \\
\hline DNR status & $5(2 \%)$ \\
\hline Chemotherapy use (within 30 days) & $51(20 \%)$ \\
\hline Radiotherapy use (within 90 days) & $4(2 \%)$ \\
\hline Weight loss $>10 \%$ within 6 months & $79(31 \%)$ \\
\hline Steroid use & $17(7 \%)$ \\
\hline Ascites & $161(63 \%)$ \\
\hline Bleeding disorder & $4(2 \%)$ \\
\hline Dyspnea at rest & $8(3 \%)$ \\
\hline Impaired sensorium & $5(2 \%)$ \\
\hline Pneumonia & $5(2 \%)$ \\
\hline Sepsis & $30(12 \%)$ \\
\hline \multicolumn{2}{|l|}{ ECOG status } \\
\hline 0 & $2(1 \%)$ \\
\hline 1 & $141(56 \%)$ \\
\hline 2 & 100 (39\%) \\
\hline 3 & $11(4 \%)$ \\
\hline Hematocrit (\%) (median, IQR) & $33.9(30.9-37.5)$ \\
\hline WBC $\left(\times 10^{9} / \mathrm{L}\right)$ (median, IQR) & $8.3(6.2-11.4)$ \\
\hline Albumin (g/L) (median, IQR) & $31(27-35)$ \\
\hline Creatinine ( $\mu \mathrm{mol} / \mathrm{L})$ (median, IQR) & $58(47-81)$ \\
\hline \multicolumn{2}{|l|}{ Procedure type } \\
\hline Gastrointestinal resection & $219(86 \%)$ \\
\hline Multi-visceral resection & $22(9 \%)$ \\
\hline Lysis of adhesions & $7(3 \%)$ \\
\hline \multicolumn{2}{|l|}{ Anastomoses } \\
\hline Any anastomosis & $164(65 \%)$ \\
\hline Gastro-jejunal & $17(7 \%)$ \\
\hline Small bowel-small bowel & $63(24 \%)$ \\
\hline Small bowel-large bowel & $86(34 \%)$ \\
\hline Large bowel-large bowel & $13(5 \%)$ \\
\hline \multicolumn{2}{|l|}{ Stoma } \\
\hline Any stoma & 91 (35\%) \\
\hline Gastrostomy & $4(2 \%)$ \\
\hline Jejunostomy & $4(2 \%)$ \\
\hline lleostomy & $35(14 \%)$ \\
\hline Colostomy & 46 (18\%) \\
\hline
\end{tabular}

Gl, gastrointestinal; DNR, do not resuscitate; ECOG, Eastern Cooperative Oncology Group; IQR, interquartile range; WBC, white blood cell. multivariable regression into a new logistic model. For 30-day morbidity, the simplified model had an AUC of 0.64 (95\% CI $0.51-0.77$ ) and sensitivity and specificity of 0.77 and 0.57 , respectively. For 30-day mortality, the simplified model had an AUC of 0.71 (95\% CI $0.55-0.86)$ and sensitivity and specificity of 0.59 and 0.80 , respectively. The receiver operating characteristic (ROC) plot summaries comparing UC Davis, multivariate, and simplified models for morbidity and mortality are plotted in Figure 1. The model summaries are included in Table 4.

To translate the 30-day mortality simplified model into a clinical tool for prediction, we constructed heatmaps of expected risk. The heatmap skeleton was a matrix with ECOG on one axis and preoperative albumin on the other. Preoperative albumin was stratified by rounding off the observed quartile of albumin in the cohort to the nearest $5 \mathrm{~g} / \mathrm{L}$. The average expected risk was determined for each cell of the heatmap and colored with a gradient of green to red, with green representing the lowest risk. We constructed a similar heatmap with the empirically observed mortality in our cohort, with the observed mortality aggregated and color-coded for each cell (Figure 3).

\section{DISCUSSION}

PC complicated with IO is one of the most common indications for surgical intervention among advanced cancer patients (10). The peritoneum houses intra-abdominal organs and is inevitably involved in disseminated end-stage cancer. Hence, PC represents a "common end point" of most advanced cancers, where patients may develop complex, multilevel IO and thus suffer from progressive inability to tolerate food, intractable abdominal pain, and distension $(13,14)$. Surgery usually entails bowel resection, bypass, and/or creation of a decompressive ostomy (15). Among our patients, a majority (86\%) required GI resection with a frequent need for bowel anastomoses and stoma creation. As such, palliative GI surgery when performed in PC patients represents a unique group where surgery may be extensive and associated with significant postoperative complications (16).

In one of the largest series of palliative surgical procedures, Miner et al. observed at 30-day postoperative morbidity of $29 \%$ and mortality of $11 \%$ (6). In the context of PC, a systemic review of 17 retrospective studies including 868 patients found that serious complications occurred in up to $44 \%$ of patients, while mortality ranged from $6 \%$ to $32 \%$ (5). Similarly, we found that 30 -day morbidity and mortality were $43 \%$ and $21 \%$, respectively, among PC patient who had undergone palliative GI surgery. However, while opponents of palliative surgery tend to focus on the high complications rates, many fail to acknowledge the high symptom resolution (up to $80 \%$ to $100 \%$ ) and low "symptom recurrence" rates after surgical intervention. In fact, our study revealed that none of our patients required repeated operation intervention for IO. Defining the parameters of surgical success is thus of 
TABLE 2 | Predictors of 30-day morbidity and mortality after palliative Gl surgery.

\begin{tabular}{|c|c|c|c|c|}
\hline Variable & 30-day morbidity $(n=110)$ & p-Value & 30-day mortality ( $n=53$ ) & p-Value \\
\hline Male & 42 & 0.70 & 16 & 0.11 \\
\hline Smoker & 6 & 0.34 & 1 & 0.69 \\
\hline Lung & 3 & 1 & 1 & 1 \\
\hline Stomach & 15 & 0.70 & 7 & 0.81 \\
\hline Pancreas & 8 & 0.41 & 5 & 0.17 \\
\hline Endometrial & 4 & 0.41 & 3 & 0.1 \\
\hline Cervix & 1 & 0.39 & 0 & 0.58 \\
\hline Others & 1 & 0.43 & 12 & NA \\
\hline Presence of lung metastases & 29 & 0.56 & 14 & 0.72 \\
\hline Presence of liver metastases & 35 & 1 & 20 & 0.32 \\
\hline \multicolumn{5}{|l|}{ Comorbid disease } \\
\hline Hypertension & 40 & 0.79 & 53 & 0.12 \\
\hline Congestive heart failure & 0 & 1 & 0 & 0.58 \\
\hline Peripheral vascular disease & 5 & 0.24 & 0 & 1 \\
\hline Renal failure & 2 & 1 & 1 & 1 \\
\hline Dialysis & & & 0 & 0.58 \\
\hline \multicolumn{5}{|l|}{ Preoperative clinical characteristics } \\
\hline Emergency Surgery & 69 & 0.18 & 38 & 0.51 \\
\hline Prehospital Location (home) & 109 & 0.39 & 51 & 0.27 \\
\hline Independent functional status & 108 & 0.47 & 50 & 0.36 \\
\hline DNR status & 1 & 0.39 & 1 & 1 \\
\hline Chemotherapy use (within 30 days) & 24 & 0.64 & 9 & 0.69 \\
\hline Radiotherapy use (within 90 days) & 1 & 0.64 & 1 & 1 \\
\hline Weight loss $>10 \%$ within 6 months & 33 & 0.79 & 17 & 0.86 \\
\hline Steroid use & 13 & 0.005 & 3 & 1 \\
\hline 2 & 51 & & 26 & \\
\hline 3 & 5 & & 5 & \\
\hline Hematocrit (\%) (median, IQR) & $32.7(29.7-36.1)$ & 0.003 & $33.8(30.0-37.1)$ & 0.23 \\
\hline WBC (×109/L) (median, IQR) & $8.8(6.5-12.1)$ & 0.14 & $8.8(6.3-12.1)$ & 0.2 \\
\hline Albumin (g/L) (median, IQR) & $29.5(26-34)$ & 0.003 & $28(24-32)$ & $<0.001$ \\
\hline Creatinine ( $\mu \mathrm{mol} / \mathrm{L})$ (median, IQR) & $56(43-76)$ & 0.58 & $55(45-73)$ & 0.94 \\
\hline \multicolumn{5}{|l|}{ Procedure type } \\
\hline Gastrointestinal resection & 96 & 0.85 & 49 & 0.02 \\
\hline Multi-visceral resection & 10 & & 0 & NA \\
\hline Lysis of adhesions & 2 & & 2 & \\
\hline \multicolumn{5}{|l|}{ Anastomoses } \\
\hline Any anastomosis & 66 & 0.19 & 29 & 0.1 \\
\hline Gastro-jejunal & 6 & 0.61 & 5 & 0.36 \\
\hline Small bowel-small bowel & 30 & 0.46 & 10 & 0.28 \\
\hline Small bowel-large bowel & 33 & 0.29 & 17 & 0.87 \\
\hline Large bowel-large bowel & 6 & 1 & 2 & 1 \\
\hline \multicolumn{5}{|l|}{ Stoma } \\
\hline Any stoma & 44 & 0.24 & 23 & 0.2 \\
\hline Gastrostomy & 3 & 0.32 & 2 & 0.19 \\
\hline Jejunostomy & 4 & 0.03 & 2 & 0.19 \\
\hline Ileostomy & 19 & 0.20 & 5 & 0.37 \\
\hline Colostomy & 19 & 0.87 & 11 & 0.55 \\
\hline
\end{tabular}

Gl, gastrointestinal; DNR, do not resuscitate; ECOG, Eastern Cooperative Oncology Group; IQR, interquartile range; WBC, white blood cell; NA, Not applicable. Values in bold indicate $p<0.05$. 
A

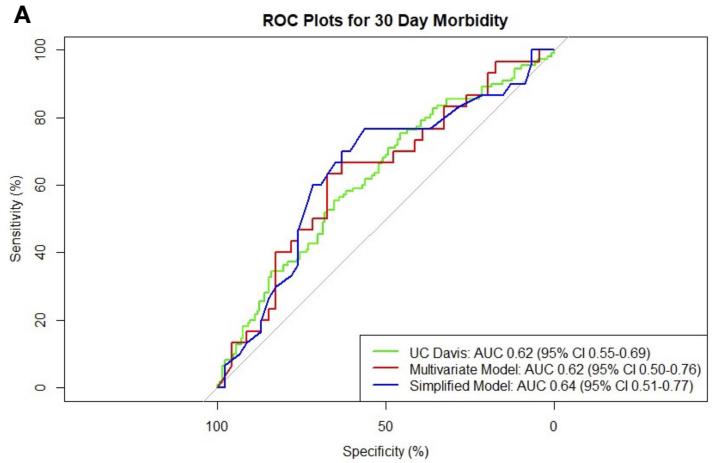

B

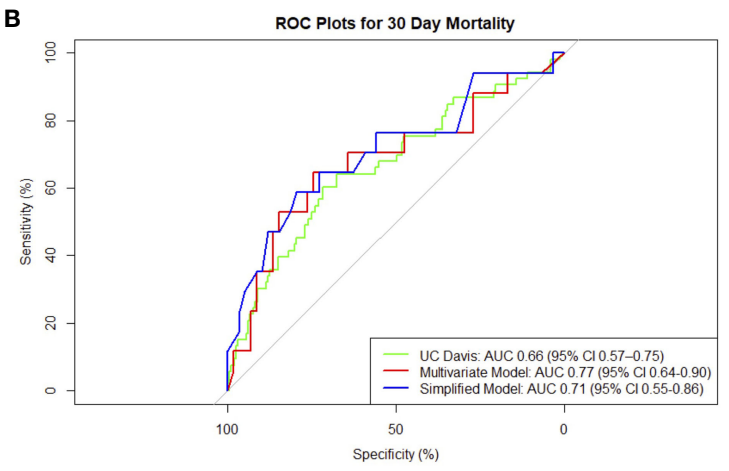

FIGURE 1 | Composite receiver operating characteristic (ROC) plots for 30-day (A) morbidity and (B) mortality models.

TABLE 3 | Model summary of multivariable model for 30-day mortality.

Site of primary malignancy

Ovary

Endometrial

Preoperative clinical characteristics

DNR status

Impaired sensorium

Sepsis

ECOG status

WBC

Albumin

$$
\begin{gathered}
0.20(0.01-1.07) \\
0(0-999) \\
0(0-999) \\
0(0-999) \\
0.20(0.02-1.03) \\
1.59(1.07-2.38) \\
1.50(0.98-2.33) \\
0.60(0.39-0.89)
\end{gathered}
$$

0.13

DNR, do not resuscitate; ECOG, Eastern Cooperative Oncology Group; WBC, white blood cell. Values in bold indicate $p<0.05$.

A

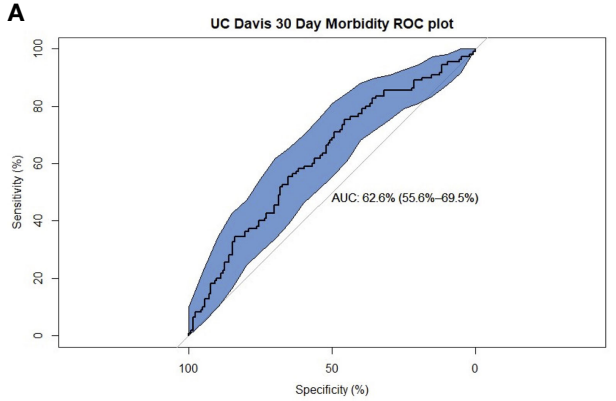

C

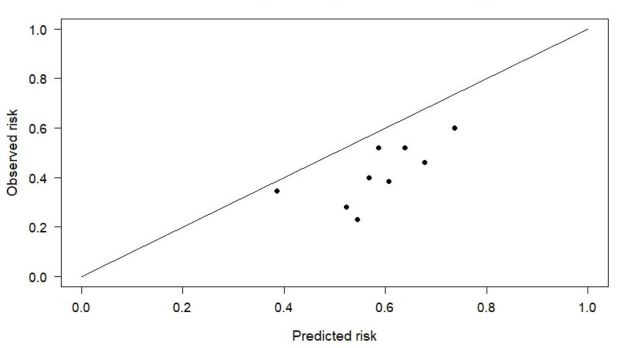

B

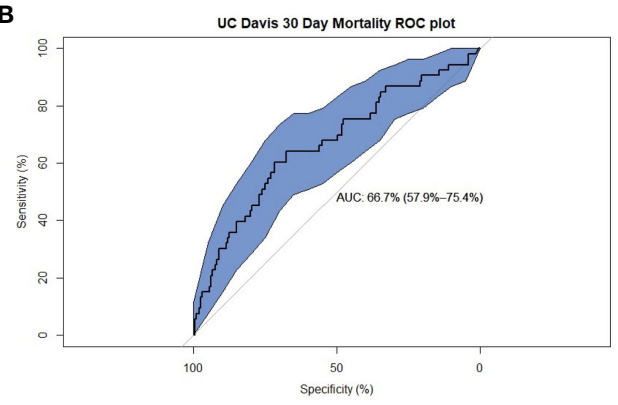

D

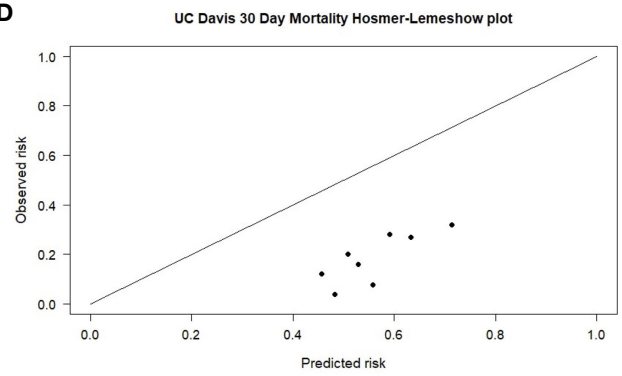

FIGURE 2 | Receiver operating characteristic (ROC) plots for UC Davis predictions of 30-day (A) morbidity and (B) mortality. Hosmer-Lemeshow (H-L) plots for UC Davis predictions of 30-day (C) morbidity and (D) mortality. 
TABLE 4 | Model summary of simplified models.

\begin{tabular}{lcc}
\hline Variable & Adjusted odds ratio (95\% Cl) & p-Value \\
\hline 30-Day morbidity & & \\
ECOG status & $1.26(0.94-1.71)$ & 0.12 \\
Albumin & $0.71(0.52-0.97)$ & $\mathbf{0 . 0 3}$ \\
30-Day mortality & & $\mathbf{0 . 0 1 8}$ \\
ECOG status & $1.56(1.08-2.26)$ & $\mathbf{0 . 0 1 2}$ \\
Albumin & $0.60(0.40-0.89)$ &
\end{tabular}

ECOG, Eastern Cooperative Oncology Group.

Values in bold indicate $p<0.05$.

paramount importance-a patient's morbidity or mortality shortly after palliative surgery should not constitute a failure if the wishes of the patients were fulfilled and they had enjoyed a "good" end-of-life experience from their perspective with adequate symptom resolution.

At the time of consideration of palliative surgery among advanced PC patients, the prognosis is often dismal, and predicted survival is less than 1 year. Therefore, it was not surprising that our reported median survival was 109 days and $80 \%$ had demised within 1 year after surgery. The importance of identifying factors predictive of short-term mortality is essential to select patients who will benefit the most from palliative surgical interventions. While the existing UC Davis nomogram is comprehensive and useful in predicting 30-day surgical morbidity and mortality, its direct application among PC patients undergoing extensive GI surgery is questionable. The UC Davis cohort is composed of patients who had gone through a variety of surgical procedures, such as vascular, skin and soft tissue, hepatobiliary, and GI interventions. This is distinct from a PC cohort, as the extent of gut manipulation and anastomoses often results in higher rates of perioperative morbidity and mortality. Therefore, when applied to our PC patients, AUC was found to be less than 0.7, representing poor discriminatory power for both 30-day mortality and morbidity outcomes.

Hence, there was a need to devise a risk model that was more applicable to PC patients undergoing palliative GI surgery. Our model comprising ECOG status and serum albumin was found to achieve superior predictive power over the UC Davis model. As such, we advocate the use of this simplified model and translated heatmap as quick clinical tools to aid operative risk discussion.

The impact of preoperative albumin levels on outcomes suggests a role for optimization through preoperative parenteral nutrition in selected PC patients planned for palliative GI surgery. While enteral nutrition has been found to be superior to total parenteral nutrition (TPN) in improving outcomes prior to surgery, this is often not possible in the PC cohort due to multilevel IO (17). In Crohn's disease, characterized by gut failure, not dissimilar to PC patients, TPN given 60 days before major abdominal GI surgery resulted in reduced rates of postoperative complications (18). As such, it is possible that a trial of preoperative TPN can improve albumin levels and lead to improved outcomes among palliative PC patients who do not present with surgical emergencies.

A limitation of this analysis is its inability to account for patients who might have been eligible for palliative surgery but were not operated on because of other factors such as patient decisions or surgeon assessment. We note that our cohort had very few ECOG 0 or 3 patients. Patients may not have been operated on because they were either deemed good candidates for further conservative management (such as ECOG 0 patients, who may have had resolution of obstruction with further nonoperative management) or too poor candidates for surgical management (such as ECOG 3 patients). This can result in paradoxical results, which may be seen when the predicted and observed heatmaps are compared for 30-day mortality. In ECOG 3 patients, those with high albumin $>35 \mathrm{~g} / \mathrm{L}$ experienced lower than predicted risks, while no patients with moderate albumin levels of 25-30 g/L were operated on. Bias arising from surgeon selection of perceived good candidates for surgery may have caused this apparent paradox and may cause overly optimistic risk estimates (such as in the case of the UC Davis model). Further refinement of risk estimates may be possible if further data are collected on such marginal patients.
A

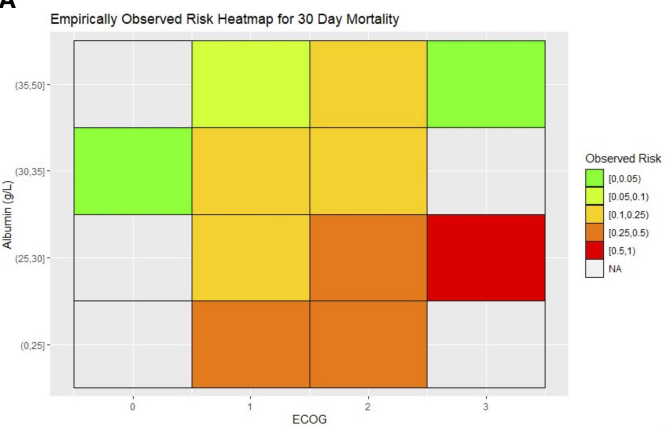

B

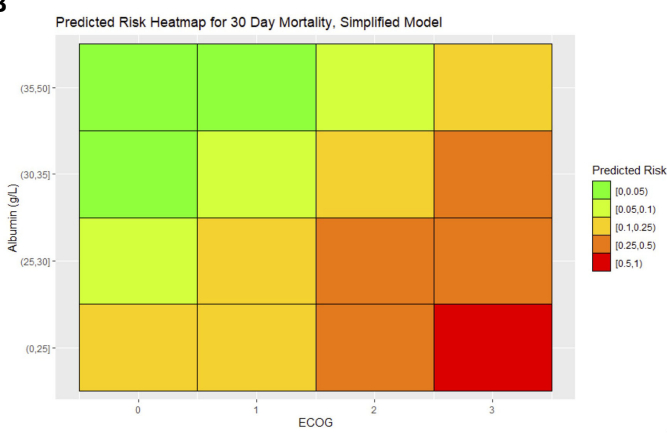

FIGURE 3 | (A) Empirically observed and (B) predicted risk heatmaps for 30-day mortality. 
In addition, the authors believe that traditional factors used to evaluate surgical efficacy such as the abovementioned postoperative complications and survival fall short of measuring outcomes most meaningful to advanced cancer patients during end of life. Survival reported in quantitative terms without reporting its quality does not attest to overall patient experience and incentivizes surgeons and non-surgeons alike to adopt measures that prolong rather than improve life. Instead, future studies should evaluate measures of quality of life, functional independence, and freedom from symptoms after surgery, which are both clinically important and meaningful to advanced cancer patients undergoing palliative surgery. Therefore, the true "value" of palliative surgery should be considered based on the preferences, expectations, and goals of care of each patient nearing end of life (19).

In conclusion, we found that a good ECOG status and high preoperative albumin levels were independently associated with good short-term outcomes after palliative GI surgery. The UC Davis nomogram showed poor performance in our cohort for the prediction of both mortality and morbidity in our patient cohort. We propose that our simplified 30-day mortality risk model and heatmap may be used as a quick stratification tool for surgeons discussing potential operative risks with patients and that further research will be needed to develop a similar tool for 30-day morbidity.

\section{DATA AVAILABILITY STATEMENT}

The original contributions presented in the study are included in the article/supplementary material. Further inquiries can be directed to the corresponding author.

\section{REFERENCES}

1. Capobianco A, Cottone L, Monno A, Manfredi AA, Rovere-Querini P. The Peritoneum: Healing, Immunity, and Diseases. J Pathol (2017) 243:137-47. doi: $10.1002 /$ path.4942

2. Flanagan M, Solon J, Chang KH, Deady S, Moran B, Cahill R, et al. Peritoneal Metastases From Extra-Abdominal Cancer - A Population-Based Study. Eur J Surg Oncol (2018) 44:1811-7. doi: 10.1016/j.ejso.2018.07.049

3. Feuer DJ, Broadley KE, Shepherd JH, Barton DP. Surgery for the Resolution of Symptoms in Malignant Bowel Obstruction in Advanced Gynaecological and Gastrointestinal Cancer. Cochrane Database Syst Rev (2000) 4:CD002764. doi: 10.1002/14651858.CD002764

4. Krouse RS, Nelson RA, Farrell BR, Grube B, Juarez G, Wagman LD, et al. Surgical Palliation at a Cancer Center: Incidence and Outcomes. Arch Surg (2001) 136:773-8. doi: 10.1001/archsurg.136.7.773

5. Paul Olson TJ, Pinkerton C, Brasel KJ, Schwarze ML. Palliative Surgery for Malignant Bowel Obstruction From Carcinomatosis: A Systematic Review. JAMA Surg (2014) 149:383-92. doi: 10.1001/jamasurg.2013.4059

6. Miner TJ, Brennan MF. Jaques DP. A Prospective, Symptom Related, Outcomes Analysis of 1022 Palliative Procedures for Advanced Cancer. Ann Surg (2004) 240:719-726; discussion 726-727. doi: 10.1097/ 01.sla.0000141707.09312.dd

7. Bateni SB, Gingrich AA, Stewart SL, Meyers FJ, Bold RJ, Canter RJ. Hospital Utilization and Disposition Among Patients With Malignant Bowel Obstruction: A Population-Based Comparison of Surgical to Medical Management. BMC Cancer (2018) 18:1166. doi: 10.1186/s12885-018-5108-9

\section{ETHICS STATEMENT}

The studies involving human participants were reviewed and approved by SingHealth Centralised Institutional Review Board. The patients/participants provided their written informed consent to participate in this study.

\section{AUTHOR CONTRIBUTIONS}

Conceptualization: MCCT. Methodology: MCCT, JSMW, and SML. Validation: JSMW, SML, DYZL, CSC, GHCT, and C-AJO. Formal analysis: JSMW, SML, DYZL, CSC, GHCT, and C-AJO. Investigation: JSMW, SML, DYZL, CSC, GHCT, and C-AJO. Resources: CSC, GHCT, C-AJO, and MCCT. Data curation: JSMW and SML. Writing - original draft: JSMW, SML, and DYZL. Writing-review and editing: JSMW, SML, DYZL, CSC, GHCT, C-AJO, and MCCT. Visualization: JSMW, SML, and DYZL. Supervision: CSC, GHCT, C-AJO, and MCCT. Project administration: CSC, GHCT, C-AJO, and MCCT. Funding acquisition: JSMW, CSC, C-AJO, and MCCT. All authors contributed to the article and approved the submitted version.

\section{FUNDING}

This study is supported by the NCCS Cancer Fund (Research) and SingHealth Duke-NUS Academic Medicine Centre, facilitated by the Joint Office of Academic Medicine (JOAM). C-AJO is supported by the National Research Council Transition Award (NMRC/TA/0061/2017). All the funding sources had no role in the study design, data interpretation, or writing of the manuscript.

8. Berger J, Lester P, Rodrigues L. Medical Therapy of Malignant Bowel Obstruction With Octreotide, Dexamethasone, and Metoclopramide. Am J Hosp Palliat Care (2016) 33:407-10. doi: 10.1177/ 1049909115569047

9. Tseng WH, Yang X, Wang H, Martinez SR, Chen SL, Meyers FJ, et al. Nomogram to Predict Risk of 30-Day Morbidity and Mortality for Patients With Disseminated Malignancy Undergoing Surgical Intervention. Ann Surg (2011) 254:333-8. doi: 10.1097/SLA.0b013e 31822513ed

10. Coccolini F, Gheza F, Lotti M, Virzì S, Iusco D, Ghermandi C, et al. Peritoneal Carcinomatosis. World J Gastroenterol (2013) 19:6979-94. doi: 10.3748/ wjg.v19.i41.6979

11. Khuri SF, Daley J, Henderson W, Hur K, Demakis J, Aust JB, et al. The Department of Veterans Affairs' NSQIP: The First National, Validated, Outcome-Based, Risk-Adjusted, and Peer-Controlled Program for the Measurement and Enhancement of the Quality of Surgical Care. National VA Surgical Quality Improvement Program. Ann Surg (1998) 228:491-507. doi: 10.1097/00000658-199810000-00006

12. Dindo D, Demartines N, Clavien P-A. Classification of Surgical Complications: A New Proposal With Evaluation in a Cohort of 6336 Patients and Results of a Survey. Ann Surg (2004) 240:205-13. doi: 10.1097/01.sla.0000133083.54934.ae

13. Chakraborty A, Selby D, Gardiner K, Myers J, Moravan V, Wright F. Malignant Bowel Obstruction: Natural History of a Heterogeneous Patient Population Followed Prospectively Over Two Years. J Pain Symptom Manage (2011) 41:412-20. doi: 10.1016/j.jpainsymman.2010.05.007 
14. Gwilliam B, Bailey C. The Nature of Terminal Malignant Bowel Obstruction and its Impact on Patients With Advanced Cancer. Int J Palliat Nurs (2001) 7:474-81. doi: 10.12968/ijpn.2001.7.10.9904

15. Helyer L, Easson AM. Surgical Approaches to Malignant Bowel Obstruction. J Support Oncol (2008) 6:105-13.

16. de Boer NL, Hagemans JAW, Schultze BTA, Brandt-Kerkhof ARM, Madsen EVE, Verhoef C, et al. Acute Malignant Obstruction in Patients With Peritoneal Carcinomatosis: The Role of Palliative Surgery. Eur J Surg Oncol (2019) 45:389-93. doi: 10.1016/j.ejso.2018.12.015

17. Bleicher J, Lambert LA. A Palliative Approach to Management of Peritoneal Carcinomatosis and Malignant Ascites. Surg Oncol Clinics North Am (2021) 30:475-90. doi: 10.1016/j.soc.2021.02.004

18. Ayoub F, Kamel AY, Ouni A, Chaudhry N, Ader Y, Tan S, et al. Pre-Operative Total Parenteral Nutrition Improves Post-Operative Outcomes in a Subset of Crohn's Disease Patients Undergoing Major Abdominal Surgery. Gastroenterol Rep (2019) 7:107-14. doi: 10.1093/gastro/goy033

19. Cohen JT, Miner TJ. Patient Selection in Palliative Surgery: Defining Value. J Surg Oncol (2019) 120:35-44. doi: 10.1002/jso.25512
Conflict of Interest: The authors declare that the research was conducted in the absence of any commercial or financial relationships that could be construed as a potential conflict of interest.

Publisher's Note: All claims expressed in this article are solely those of the authors and do not necessarily represent those of their affiliated organizations, or those of the publisher, the editors and the reviewers. Any product that may be evaluated in this article, or claim that may be made by its manufacturer, is not guaranteed or endorsed by the publisher.

Copyright (c) 2022 Wong, Lek, Lim, Chia, Tan, Ong and Teo. This is an open-access article distributed under the terms of the Creative Commons Attribution License (CC BY). The use, distribution or reproduction in other forums is permitted, provided the original author(s) and the copyright owner(s) are credited and that the original publication in this journal is cited, in accordance with accepted academic practice. No use, distribution or reproduction is permitted which does not comply with these terms. 